\title{
marges Marges
}

revue d'art contemporain Revue d'art contemporain

13 | 2011

Langage(s) de l'œuvre et de l'art

\section{Façons de parler : l'anecdote comme approche de l'œuvre}

Ways of Talking: the Anecdote as a Way of Approaching the Artwork

\section{Yaël Kreplak}

\section{(2) OpenEdition}

\section{Journals}

Édition électronique

URL : http://journals.openedition.org/marges/436

DOI : $10.4000 /$ marges. 436

ISSN : 2416-8742

\section{Éditeur}

Presses universitaires de Vincennes

\section{Édition imprimée}

Date de publication : 1 septembre 2011

Pagination : 91-106

ISBN : 978-2-84292-343-3

ISSN : $1767-7114$

\section{Référence électronique}

Yaël Kreplak, « Façons de parler : l'anecdote comme approche de l'œuvre », Marges [En ligne],

13 | 2011, mis en ligne le 15 septembre 2012, consulté le 30 avril 2019. URL : http://

journals.openedition.org/marges/436 ; DOI : 10.4000/marges.436 


\section{Façons de parler: l'anecdote comme approche de l'œuvre}

Que l'œuvre nous parle, que l'artiste ait voulu nous dire quelque chose, ou encore que l'exposition soit envisagée comme un dispositif communicationnel signifiant, ces différentes expressions attestent selon nous de la prégnance d'un modèle langagier dans la relation esthétique. Notre propos ne sera pas de discuter la pertinence d'une telle conception de l'art « comme » communication, mais d'en déplacer quelque peu l'acception habituelle, en faisant un pas de côté de la théorie vers l'empirie. Nous nous intéressons en effet non pas à ce que l'œuvre nous dit hypothétiquement - par elle-même ou en tant que médium d'expression d'un artiste ou d'un commissaire -, mais à ce que nous, nous en disons effectivement: en prenant pour objet d'investigation les discours ordinaires produits face aux œuvres et/ ou sur les œuvres, nous espérons ainsi contribuer, d'un point de vue linguistique, à une réflexion sur les propriétés de la relation esthétique et sur le rôle constitutif qu'y joue la dimension langagière.

Parler d'une œuvre est une activité qui prend assurément différentes formes, selon le locuteur (artiste, commissaire, monteur ou spectateur), le mode d'expression (écrit, oral, voire gestuel), le contexte (conférence, visite guidée, sortie entre amis), et qui peut avoir différents enjeux (rédaction d'un article critique, description pour une vente aux enchères, conversation mondaine). Notre travail de terrain a consisté à suivre, sur un mode ethnographique, une exposition d'art contemporain de sa conception à sa pratique, et à enregistrer différentes situations ordinaires de la vie de l'exposition, ce qui nous a permis de constituer un corpus diversifié pour penser les modalités de cette activité discursive. Pour préciser ce questionnement, nous avons choisi de nous focaliser ici sur un type de discours particulier, l'anecdote. Définie comme un récit bref, relatant généralement des 
/1 Voir Erving Goffman, Façons de parler,

(trad. fr. A. Kihm), Paris, Minuit, 1987

(éd. originale : Forms of Talk, Philadelphia, University of Pennsylvannia Press, 1981). faits d'importance secondaire, et idéalement divertissant, l'anecdote est une pratique ordinaire, à laquelle on a fréquemment recours dans la conversation, mais aussi dans des contextes plus institutionnels, comme le rappelle par exemple Goffman à propos de la conférence, où l'anecdote joue un rôle dans la captatio benevolentiae de l'auditoire/1. Ainsi définie, elle semble donc ne pouvoir relever que de l'« anecdotique »: participant de l'habillage d'un discours, mais ne comportant aucun des éléments déterminants qui font la " moëlle» d'un véritable propos sur l'art. Pourtant, ce type de production discursive nous a semblé intéressant à plusieurs titres. Pratique routinière, elle est commune aux experts et aux profanes, qui y ont recours dans des situations variées: c'est donc un objet public, observable, dont on peut questionner les enjeux. Dans quels contextes et à qui raconte-t-on une anecdote? Comment l'anecdote construit-elle son objet et que nous apprend-elle sur la façon qu'a son locuteur de l'aborder? Quel type de représentation de l'œuvre produit-elle et quels en sont les usages?

Pour répondre à ces différents questionnements, il conviendra tout d'abord de préciser dans quel cadre théorique et méthodologique s'inscrit ce travail, en le resituant dans la double perspective de la linguistique interactionnelle et de l'esthétique pragmatiste. Dans un deuxième temps, on commentera brièvement des extraits mettant en jeu différents types d'anecdotes. Enfin, ces réflexions nous permettront de faire le lien avec un projet d'exposition, qui à bien des égards a impulsé cette réflexion, et qui porte spécifiquement sur ces « récits ordinaires », dont il questionne les modalités de recueil, de circulation et d'exposition.

\section{L'anecdote comme pratique conversationnelle et usage de l'œuvre}

\section{Définir un point de vue linguistique}

Notre corpus de travail est constitué d'enregistrements vidéo réalisés dans un centre d'art contemporain et lors de visites commentées dans différentes institutions. Notre recherche s'inscrit dans le champ de l'analyse conversationnelle: s'il n'est pas lieu de rendre compte ici de tous les enjeux d'un tel positionnement, on en rappellera néanmoins brièvement à des fins de clarification les principaux postulats. L'analyse conversationnelle, qui s'est développée dans les années 1960 aux États-Unis, a pour objet l'analyse des activités langagières, en ce qu'elles rendent intelligibles l'organisation localement accomplie d'une interaction. La conversation est constituée en objet prototypique, où se jouent continuellement la négociation non seulement 
du sens et de l'organisation thématique de l'échange, mais aussi la négociation de la participation, par les ajustements des personnes présentes les unes aux autres et leurs changements de statut (alternances de tour et de position locuteur/auditeur). Par l'analyse d'enregistrements audio-vidéo, transcrits de manière détaillée, les travaux conversationnalistes mettent en évidence les dimensions radicalement contextuelle, émergente et incarnée du langage/2.

Un tel angle d'approche a des conséquences d'au moins deux ordres en ce qui concerne notre objet. D'une part, l'analyse conversationnelle vise, dans ses descriptions de l'interaction, à restituer la perspective des locuteurs. Méthodologiquement, cela implique d'utiliser les données naturelles non pas pour vérifier que l'anecdote est bien « un récit bref, relatant des faits secondaires et divertissants »- soit selon une détermination a priori -, mais au contraire, pour voir ce que les participants définissent comme anecdote en la produisant/l'écoutant, par l'identification de pratiques récurrentes et structurantes. Cela permet ainsi de définir l'anecdote par ses usages, et de repenser par là sa finalité possible. D'autre part, une telle approche postule que c'est collaborativement que se construisent les objets d'attention communs. Cette collaboration est bien sûr d'ordre verbal (parler de la même chose), mais elle passe aussi par des ressources visuelles (regarder son interlocuteur), posturales (manifester son écoute par une position spécifique), gestuelles ou artefactuelles (manipuler des objets présents en relation avec l'anecdote/3) - soit autant d'éléments qui vont dans le sens d'une conception non logocentrée de l'interaction. De la littérature abondante qui traite de cette conception dite «multimodale » du langage, on ne retiendra ici que les travaux de Christian Heath et Dirk vom Lenh qui portent sur les interactions dans l'espace muséal/4. Leurs recherches sur les ajustements des visiteurs les uns par rapport aux autres et par rapport aux œuvres dans différents espaces d'exposition ont montré comment s'organise séquentiellement le passage d'un focus d'attention à un autre, et en quoi le fait de rendre publics (verbalement, mais aussi gestuellement et spatialement) ses modes d'observation contribue à établir une perception, et par là possiblement une interprétation, commune d'une œuvre. On adoptera ainsi à leur suite cette idée, centrale pour nos recherches, que c'est socialement, interactionnellement et réflexivement, que se construit le sens d'une œuvre/5.

\section{Esthétique pragmatiste: penser les usages de l'œuvre}

Une telle approche du langage en général, et les conclusions de certains travaux portant sur l'espace muséal en particulier, nous semblent entrer directement en résonance avec la conception prag-
/2 Pour plus de précision, voir Elisabeth Gülich et Lorenza Mondada, « Konversationanalyse », dans Günter Holtus, Michael Metzelin et Christian Schmitt (sld.), Lexikon der Romanistischen Linguistik, Vol. Band I, 2, Tübingen, Max Niemeyer, 2001, p. 196-250.

/3 Les travaux conversationnalistes sur la question de la narration ont ainsi notamment mis en évidence le rôle de l'auditeur dans la structuration du récit, par la production de ratifications (mimiques, hochements de têtes...) qui orientent le narrateur vers certains développements ou, au contraire, l'invitent à modifier le rythme de son récit, voire à céder son tour. Voir Charles Goodwin, « Audience diversity, participation and interpretation ", dans Text 6 (3), 1986, p. 283-316.

/4 Dirk vom Lehn, Christian Heath et alii, " Exhibiting Interaction: Conduct and Collaboration in Museums and Galleries », Symbolic Interaction $\mathrm{n}^{\circ} 24$ (2), 2001, p. 189-216. Voir aussi Dirk vom Lehn, "The Body as Interactive Display: Examining Bodies in a Public Exhibition ", Sociology of Health \& Illness, vol. 28, $n^{\circ} 2$, 2006, p. 223-251.

/5 Leurs travaux visent à observer « la manière dont les gens [...] constituent de manière réflexive le sens et 
la signifiance des objets et artefacts et la manière dont ces caractéristiques matérielles informe de manière réflexive la production et l'intelligibilité de la conduite et de l'interaction ", Christian Heath et al., « Crafting participation: designing ecologies, configuring experience ", Visual Communication, Vol.1 (1), 2002, p. 9-34.

/6 Jean-Pierre Cometti, Qu'est-ce que le pragmatisme?, Paris, Folio, 2010, p. 239.

/7 Louis Quéré, " Le public comme forme et modalité d'expérience ", dans Daniel Céfaï et Dominique Pasquier (sld), Les Sens du public, Paris, PUF, 2002, p. 112-134, p. 118.

/8 ibid.

/9 Voir les critiques adressées par Roger Pouivet à l'esthétique pragmatiste, Qu'est-ce qu'une œuvre d'art?, Paris, Vrin, 2007, p. 31.

/10 L'analyse conversationnelle emploie le terme de " collection » pour renvoyer à la constitution d'un ensemble de cas analysables dans la même perspective. matiste de l'expérience esthétique, telle que l'a définie Jean-Pierre Cometti: une esthétique qui, à la suite de l'ouvrage fondateur de John Dewey, L'Art comme expérience, n'ignore pas «les rapports qui relient les œuvres à un contexte de croyances, de discours et d'activités socialisées/6 $\mathbf{~ » . ~ S a n s ~ v o u l o i r ~ e n ~ a m o i n d r i r ~ l a ~ p o r t e ́ e , ~ m a i s ~}$ à rebours de toute idéalisation romantique, il faut saisir l'expérience esthétique dans la continuité des expériences ordinaires et l'inscrire dans la vie sociale d'une communauté spécifique, le "public d'art", qui se définit précisément "en termes d'expérience partagée" comme le rappelle Louis Quéré/7. Ce que fait collectivement le public dans la visite d'une exposition, "c'est parachever une configuration », dans laquelle le public est " partie prenante de la réalisation de l'œuvre », dans la mesure où «le sens d'une œuvre réside pour une grande part dans les effets de tous ordres qu'elle produit à travers la réception/8 ». Le corrélat de cette primauté accordée à l'expérience, en tant qu'événement social, public et partageable, est ainsi de concevoir l'œuvre non comme un objet clos, immuable, mais au contraire, comme un artefact infiniment mobilisable et modulable dans l'interaction. Précisons toutefois qu'il ne s'agit pas de refuser la reconnaissance de propriétés propres à l'œuvre d'art, mais simplement de ne pas poser le problème en termes essentialisants ou ontologiques/9, pour se focaliser sur les pratiques autour des œuvres - dont l'activité discursive qui nous intéresse ici constitue une bonne partie.

Ce rapide cadrage nous a permis d'éclairer notre conception de la relation esthétique comme configuration réciproque et socialement située. L'anecdote nous intéressera donc désormais en tant que mode d'organisation et de structuration d'une expérience particulière, et en tant que modalité de représentation contextuelle, à toutes fins pratiques, d'une œuvre ou d'un savoir à son propos. Pratique routinière, l'anecdote met-elle en jeu des savoirs « ordinaires » - et si oui, de quel ordre sont-ils? Que peut-elle dire de l'œuvre et du public d'art qui l'observe? L'analyse empirique de brefs extraits nous permettra d'apporter quelques éléments de réponse.

\section{L'anecdote, un objet empirique}

\section{Perspectives d'analyse}

S’il n'est pas lieu de développer pleinement ici une réflexion de type conversationnaliste, on souhaiterait dans cette section esquisser des perspectives analytiques, afin d'exemplifier les remarques qui précèdent. Pour ce faire, nous avons constitué une petite collection/10 d'extraits d'anecdotes à propos d'œuvres. Bien qu'elles aient des 
formes et usages variables, car adaptés à leur situation d'énonciation, elles présentent néanmoins des pratiques récurrentes et des propriétés communes, qui justifient leur rapprochement ici. Dans le premier extrait/11, enregistré lors d'une réunion de conception d'une exposition, un artiste (Johann) raconte à des membres du centre d'art (Lila et Pauline) comment il a conçu ses dernières pièces, dont l'idée lui est venue à la suite d'un quiproquo lors d'une précédente exposition. Le deuxième extrait met en scène un médiateur (Arnaud) commentant à un groupe une série de photographies et recourant pour cela à la narration de visites précédentes, rapportant les remarques formulées par certains visiteurs. Enfin, le troisième extrait a été enregistré lors du montage de son installation par un artiste (Marc), interrompu dans son travail par un autre artiste (Mike) venant lui parler d'une œuvre faisant écho à la sienne. Ces extraits permettent de voir comment émerge la narration d'une anecdote dans un cours d'activité, et comment un tour de parole se constitue en tant que « raconter quelque chose à propos d'une œuvre »: il s'agit alors de voir en quoi l'anecdote trouve sa signification et son usage par ce qu'elle fait et permet de faire à un moment précis de l'interaction. À des fins pratiques, on commencera par mettre en évidence les propriétés formelles, analysables dans nos extraits, de l'activité narrative. L'identification d'un récit passe tout d'abord par la reconnaissance de ses frontières, telles qu'elles émergent dans la structuration du tour de parole. En ouverture, on relève ainsi généralement un connecteur fort (d'ailleurs, donc, alors), encadré ou non de courtes pauses, et précédant la « préface » du récit:

Exemple 1, white cube

1 JOH D'Allleurs c'est ça qu' est super drôle c'est c'qui est

2 arrivé avec euh: (0.5) cette pièce là euh: (0.7) du

3 white cube c'est encore un autre rebondiss `ment\ (0.9)

La préface a pour fonction de contextualiser le récit, en le situant dans une chronologie particulière et/ou en situant la position du narrateur. Ainsi, dans l'exemple 3, Mike, qui a été rapidement présenté à Marc quelques heures plus tôt, contextualise son propos par son origine géographique, élément qui a sa pertinence par rapport au récit:

Exemple 3, turkish artist:
1 MIK this ey: $\mathbf{i}$ come from albany in australia/
2 euh can you speak english//
3 MAR i can [i can slowly]

/11 Pour faciliter la lecture, nous avons fait figurer les transcriptions complètes de ces extraits à la fin du texte, ainsi que la convention à partir de laquelle elles ont été réalisées. Précisons que les vidéos ont été faites avec l'accord des personnes filmées et des institutions, et que les extraits sont anonymisés. 
/12 Ces éléments sont perceptibles sur la vidéo, mais pourraient faire l'objet d'une notation, selon des conventions permettant de décrire finement la coordination du geste, de la posture, du regard et de la parole. Voir [http://icar. univ-lyon2.fr/ecole_ thematique/tranal_i/ documents/Mosaic/ICAR_ Conventions_ICOR.pdf]
4 MIK

[(inaud.) ] so i come from albany in australial originally but not from a long ${ }^{\circ}$ time $\mathrm{a}\left[\mathrm{go}^{\circ} \backslash(\right.$.$) but (this ; there's$

Cette première séquence a donc une double fonction: à la fois ouvrir le récit, et exhiber cette ouverture aux participants. On remarque en effet que, dans l'extrait 1 par exemple, Lila et Pauline identifient comme récit le tour de Johann, par leurs ratifications (hochements de tête, murmures d'approbation) et leur position d'écoute attentive/12. Le déploiement du récit, quant à lui, est variable: plus ou moins long, plus ou moins fluide. Autant le récit des propos des visiteurs dans l'extrait 2 est rapide, occupant une simple unité de tour, autant la construction progressive de celui de Johann est lisible dans l'adjonction de segments explicatifs supplémentaires, l'utilisation du catalogue comme ressource pour partager l'objet auquel il est fait référence, etc. Ce déroulement a une incidence sur la visibilité de la fin du récit, en fonction des modalités de son intégration au tour (dans le prolongement immédiat ou après les interventions des autres participants). De fait, la fin du récit est constituée réciproquement dans la façon qu'ont les auditeurs de réagir, selon qu'ils prennent très rapidement la parole (voire interrompent presque le tour précédent):

Exemple 2, hamilton:

4 ARN alors (1.3) hier j'ai eu un groupe un peu plus âgé qui:

5 en discu-c'est vrai qu'à l'époque (.) ça nous choquait

6 pas et maint'nant on est plus:

7 JEA (mais ; bah) oui [mais parce que l'information n'était\&

8 ELI [bah oui

ou qu'ils participent de manière minimale, relançant l'activité narrative:

Exemple 1, white cube:

$29 \mathrm{JOH}$ mais enfin enfin là c'est encore des histoires de de de

30 mauvaise (o.6) de mauvaise (.) de mauvaise traduction

$31 \quad{ }^{\circ}\left({ }^{\prime}\right.$ fin) de quelque chose ${ }^{\circ}[(0.6)$ mais c'est c- en fait\&

32 LIL [ouais:

33 PAU

[mmmh

$34 \mathrm{JOH}$ \& c'est vraiment $\mathrm{c}-\mathrm{c}^{\prime}$ qui m'intéresse le plus quoi c'est comme la pièce avec le: (0.4) ${ }^{\circ} l e:^{\circ}$ (une) les deux 
ou encore qu'ils gardent le silence, occasionnant un blanc dans l'échange:

Exemple 3, turkish artist:

17 MIK have to: (.) to show it to you ' ${ }^{\circ}$ cause it's

18 really ${ }^{\circ}$

$19 \quad(2,5)$

\section{MIK no it's great}

On dira donc que formellement, une anecdote émerge comme un tour plus ou moins prolongé d'un locuteur, borné à l'ouverture par une préface et à la clôture par une chute ou un segment conclusif (généralement une évaluation), qui peut faire le lien avec l'échange auquel elle appartient.

Comme on l'a mentionné, l'analyse conversationnelle s'intéresse aux activités discursives saisies dans leur temporalité, selon une conception de l'interaction comme architecture émergente et collaborative. La prise en compte du positionnement séquentiel (où et comment arrive l'anecdote dans l'échange) est donc cruciale. Dans le deuxième extrait, qui démarre après une longue phase de description des photos présentes, la narration intervient à la ligne 4 comme une (auto-) réponse à la question énoncée précédemment:

Exemple 2, hamilton:

1 ARN ${ }^{\circ}$ (peut-être pas celles qui sont là) ${ }^{\circ}$ et donc la question 2 posée c'est justement peut-on encore regarder après les

3 affaires dutroux et autres choses de ce genre\ (0.9)

4 alors (1.3) hier j'ai eu un groupe un peu plus âgé qui:

L'analyse du positionnement montre ainsi comment se structure l'activité en cours et comment est projeté le rôle que peut jouer la narration d'une anecdote - qui serait ici, après la description, de donner des éléments interprétatifs. On postule en effet que la séquentialité de l'échange produit des effets, aussi bien sur le plan thématique que sur le plan de la participation des différentes personnes en présence. Dans l'extrait 3, le fait que Mike interrompe Marc par sa venue dans son espace de travail et que son récit occupe quasiment les premiers tours de leur échange, pose un problème pratique à Marc, qui ne sait pas nécessairement que faire des informations qui lui ont été apportées: l'émergence de ce récit comme interruption d'un cours d'activité et comme rencontre sociale entre deux artistes n'a pas permis 
d'instaurer un cadre favorable au développement du récit - la suite de l'extrait consistant en une description par Marc de son œuvre. Le placement des tours narratifs ménage donc des usages particuliers que peuvent faire les participants du récit produit. Cette dimension est intrinsèquement liée à celle des formats de participation. En ce qui concerne l'intégration des auditeurs à la production narrative, on a déjà relevé la participation active de Lila et Pauline au récit, perceptible dans leurs ratifications, soit par des interventions minimales (voir plus haut), soit par des tours visant à compléter ou commenter le propos de Johann:

Exemple 1, white cube:

$20 \mathrm{JOH}$ pièce sur- où les photos [sont super moches avec les\&

21 PAU [avec un livre sorti

$22 \mathrm{JOH}$ \&bouquins qui manquent (0.5) à l'intérieur donc à partir

23 de ç[a j'ai fait une nouvelle pièce

24 LIL [une erreur bah oui une erreur UNE ERREUR PAREILLE ÇA 25 NE SE LOUPE PAS/=

$26 \mathrm{JOH}=<(($ en riant $))$ donc j'ai fait une nouvelle pièce après >

Dans l'extrait 2, les deux visiteuses, Jeanne et Eliane, prennent également la parole et, fait notable, la gardent relativement longtemps, ce qui invite le médiateur à négocier sa reprise de tour (voir sa première tentative en ligne 21) et occasionne ainsi une séquence d'échange prolongée:

Exemple 2, hamilton:

9 JEA \&pas la même (.) $x x$ d'inceste de viol étaient moins [(.)\& 10 ARN [oui

11 JEA \&disons (dans\& \&le domaine public) que [xx

$12 \mathrm{ELI}$

[c'est à dire que

13 (.) un regard vers un: un j- un jeune nu n'était pas

14 considéré comme un regard pervers parce que maint 'nant

15

16 ARN $=$ d'ac[cord

17 JEA [oui oui voilà=

$18 \mathrm{ELI}$ =un homme regarde une jeune femme (.) de quinze ans (.)

19 on voit tout de suite du pervers à l'époque on voyait la

20 beauté c'est tout

21 ARN mmh (.) c' qui est amusant c'est que j'ai un [:

22 ELI

['fin c'est 
24 JEA [oui

25 ARN haha $^{\circ}<\left((\right.$ en riant)) $)$ les collections en question ${ }^{\circ}>($. $)$ euh:

Précisément, l'anecdote est un format discursif qui tend à gommer l'asymétrie propre à certaines interactions et favorise donc l'échange: c'est bien à ce titre qu'elle nous intéresse, en tant que format d'expression privilégié d'un type de discours potentiellement collaboratif sur les œuvres/13.

\section{L'anecdote comme production de savoir}

Cette idée permet de faire le lien avec le dernier point que nous souhaitions évoquer, soit la question de l'expertise en jeu dans l'anecdote, et des modes de représentation ou de production d'un savoir qui y sont liés. Il nous semble qu'une analyse fine de la structuration d'une anecdote invite à nuancer toute réification des rôles et des savoirs qui y sont liés a priori. Si nos extraits mettent en scène différentes catégories d'experts (l'artiste parlant de son œuvre, le médiateur parlant d'une série de photographies, ou un artiste parlant d'une œuvre inconnue d'un autre), et corrélativement différentes catégories de profanes (les institutionnels du centre d'art, connaissant certes le travail de l'artiste mais n'ayant pas la compétence pour parler des circonstances de sa production, les visiteurs, conçus comme des pages (variablement) blanches, ou un artiste ne connaissant pas le travail apparemment proche d'un autre), on espère avoir mis en évidence que les manières de raconter peuvent occasionner différentes participations, qui peuvent être autant de contradictions ou de développements des éléments apportés. Les façons de parler - et d'écouter - jouent donc un rôle crucial dans la co-élaboration d'une version d'une œuvre, qui a sa pertinence comme représentation contextuelle, émergente et partagée. Les visiteurs de l'extrait 2 interviennent sûrement en partie parce que la narration de propos rapportés légitime leur propre intervention et, ce faisant, ils font évoluer le commentaire des photos vers la contextualisation de la démarche du photographe et celle de ses différentes réceptions possibles. C'est moins l'œuvre qui est l'objet du récit ici que les propos tenus à son sujet, mais le médiateur rend ainsi public un débat interprétatif et fait des visiteurs présents des acteurs de celui-ci, en les laissant partager leur expertise sur des facteurs d'ordre socio-historique.

Ces quelques remarques permettent donc de saisir en quoi l'anecdote, mode tenu pour mineur, a des conséquences majeures en termes d'organisation de l'interaction et de production d'un savoir ordinaire sur les œuvres. Bien que l'on ait davantage exemplifié
/13 On nuancera toutefois cette idée avec l'exemple 3, l'anecdote racontée par Mike n'étant pas du tout ressaisie par Marc, pour des raisons d'ordre interactionnel que l'on a déjà évoquées. 
/14 Ce projet est soutenu par le Centre d'art contemporain de Nice - la Villa Arson et la DRAC PACA, en partenariat avec la Kadist Art Foundation (Paris).

/15 Voir d'ailleurs son projet Anecdote Archive, qui consiste en une collection de brefs extraits vidéos de personnes (artistes, curateurs...) racontant en quelques minutes une histoire à propos d'une œuvre: [http:// anecdotearchive.org/] (consulté le 15/10/10).

/16 Cette citation est extraite d'un document de travail. ici des pratiques de production et de participation à une séquence narrative que développé ce qui est effectivement dit à propos des œuvres, il nous semble néanmoins possible de caractériser le savoir auquel on fait référence comme " ordinaire », pour plusieurs raisons intrinsèquement liées: du fait du contexte dans lequel il est énoncé bien qu'il s'agisse de contextes de travail, ils ne sont pas formels - ; de son format de production, improvisé et ne prétendant pas à la généralité ou la fixation; enfin, du fait de son caractère sélectif - ne visant pas l'exhaustivité, mais répondant à des besoins interactionnels spécifiques.

Qu'il s'agisse de raconter les circonstances de production d'une pièce, ou qu'une œuvre fasse parler d'autre chose (d'un faits divers ou d'un autre artiste), ou toutes autres circonstances non exemplifiées ici: pour rendre compte de la dimension langagière dans la relation à l'œuvre et de la façon dont la socialité configure la réception (voire la production), l'analyse conversationnelle nous semble offrir des outils particulièrement pertinents. L'anecdote constitue, dans cette perspective, un objet empirique de choix pour analyser la structuration collaborative d'un discours et ses usages possibles, qui mériterait des analyses plus développées. Ces questionnements font également l'objet de réflexions d'ordre artistique ou curatorial, dans le cadre d'un projet d'exposition auquel nous collaborons: c'est ce point que nous allons désormais développer pour réfléchir plus spécifiquement aux enjeux de ces anecdotes et à leur circulation possible dans les « mondes de l'art».

\section{De l'analyse à la production: l'anecdote comme matériau artistique}

À bien des égards, notre participation à un projet curatorial intitulé "Des récits ordinaires" a impulsé cette réflexion/14. Commissionné par Grégory Castéra, en collaboration avec David Antin (performer et poète), Joseph del Pesco (commissaire)/15 et Franck Leibovici (artiste), ce projet repose sur une définition liminaire de ces « récits ordinaires », qui en structure la conception et qu'on peut énoncer dans les termes suivants: "Les récits ordinaires sont des modalités d'existence des œuvres dont les propriétés permettent de renouveler l'interprétation des œuvres en exprimant comment œuvres et discours oraux se composent entre eux/16. ». Avec un angle différent mais prenant appui sur les pratiques incarnées pour développer une réflexion curatoriale -, une telle définition prolonge l'approche développée précédemment: de quoi rendent compte ces récits ordinaires? Quel usage peut-on en faire? En prenant acte de l'importance de 
la discursivité dans la relation esthétique, l'option développée dans ce projet d'exposition peut sembler très radicale: il s'agit en effet de postuler que les récits oraux produits en diverses circonstances non seulement participent de la vie sociale de l'œuvre, mais qu'ils en sont même une des médiations propres, au même titre qu'une reproduction photographique ou un texte de présentation. D'où ce parti pris d'exposer non pas des œuvres, dans leur matérialité, mais des récits produits à leur propos, en tant qu'ils en sont une "modalité d'existence »-ce qui pose la question de la valeur de représentativité de ces récits et de leur identification comme tels. Corrélativement, il s'agit aussi de pleinement revaloriser le statut de ces récits produits à propos de pièces, en tant qu'ils en renouvellent l'interprétation - ce qui pose la question de leurs possibles fixation, circulation et effet.

En premier lieu, une collection d'anecdotes doit être constituée. Une liste de narrateurs, pour la plupart issus des mondes de l'art, est en cours d'élaboration. Sur la base des connaissances conversationnalistes, qui ont mis en évidence la construction en archipel des séquences narratives dans une interaction (le fait qu'une anecdote en suscite une autre par exemple avec le même schéma actanciel ou la même thématique), il a pour l'instant été décidé d'organiser des dîners avec différents invités, ayant idéalement un minimum d'interconnaissance pour garantir l'effet de "normalité » du repas. Au préalable, ces invités sont prévenus d'un certain nombre de « règles du jeu ", les enjoignant minimalement à respecter l'ordinarité de la situation sociale "dîner », à ne pas évoquer lesdites règles et à placer leur anecdote au moment leur semblant opportun. Les dîners font l'objet d'un enregistrement audiovidéo intégral. Les séquences de narration seront ensuite transcrites et retravaillées pour constituer des partitions, qui seront le support d'activations variables, encore à déterminer. Ce protocole reste problématique sur certains points, en lien avec des réflexions énoncées précédemment. On retrouve notamment une tension possible entre une définition normative du récit, néanmoins nécessaire pour déterminer les modalités de participation des invités, et la part à accorder à sa production émergente lors de la rencontre sociale organisée à cette fin spécifique. Faut-il prévenir les narrateurs des enjeux et leur passer une commande d'anecdote? Qu'attend-on comme type de récit (en termes de fiction/véracité, longueur, tonalité, rapport à l'œuvre, contenu descriptif/interprétatif)? Un modèle, avec des passages obligés (développement de la description, énoncé des coordonnées contextuelles de la rencontre avec l'œuvre...), doit-il être dégagé pour qu'un récit puisse «valoir pour » une œuvre? Cette tension permet donc de prendre la mesure du lien problématique entre ordinarité et institutionnalité/17, et pose
/17 On entend « institutionnel» au sens du performatif austinien: l'énoncé « Je te baptise » n'a de sens comme baptême effectif que s'il est prononcé par le locuteur compétent dans les circonstances institutionnelles adéquates. Autrement dit, dans quelles circonstances un récit sur une œuvre vaut-il pour l'œuvre? Comment l'instituer en tant que tel? 
/18 Notamment, qui est l'artiste exposé, le narrateur ou l'auteur de l'œuvre dont il est question? Comment, corrélativement, rémunérer les différentes parties prenantes? la question des critères distinctifs des récits retenus. Ces aspects feront assurément l'objet d'une réévaluation, une fois les premiers enregistrements effectués.

L'exposition de la collection ainsi constituée donnera à voir/entendre/lire des récits valant pour une présentation de la pièce racontée, ce qui d'un point de vue institutionnel et économique est d'un intérêt certain/18, mais on s'en tiendra ici à des réflexions ayant directement trait à la question de l'anecdote. Fondée sur une démarche de singularisation - inviter une personne à raconter les circonstances subjectives de sa rencontre avec une pièce et à rendre compte de son expérience propre -, la collection procèdera par abstraction, en détachant ce récit à la fois de sa voix singulière (par le travail de transcription puis de mise en partition), et du cadre dans lequel il a été produit (l'exposition redoublant ainsi la situation de recueil, déjà détachée de l'événement narré). Ces « récits ordinaires », dont on a souligné plus haut quelques-unes des propriétés (informalité, subjectivité, sélectivité), se trouveront ainsi recontextualisés les uns par rapport aux autres dans l'exposition - soit non plus en regard d'une perspective singulière qui lui donnait son sens, mais en regard d'autres histoires saisies dans leur instantanéité -, et fonctionneront vraisemblablement comme un contexte pour d'autres récits, suscitant d'autres descriptions et interprétations, discutant celles énoncées. C'est donc bien la circulation de la parole qui se trouvera ainsi exposée: par ce processus de fixation d'une forme singulière, une telle exposition questionnera la répétition, ou dissémination, de son propre discours, dont il pourrait être intéressant, le moment venu et avec des méthodes d'enquête adéquates, d'évaluer la portée.

Pour conclure, on espère, par ces quelques remarques, avoir d'une part rendu compte de notre point de vue spécifique, soit celui d'une linguiste prenant pour objet certaines pratiques de l'art contemporain, et d'autre part avoir mis en évidence l'intérêt d'un objet « mineur » comme celui de l'anecdote pour penser la part langagière dans l'expérimentation d'une œuvre. Assurément, la parenté tant pratique que théorique entre ce projet d'exposition et notre travail de recherche a considérablement nourri cette perspective, chacun, avec ses moyens de représentation propres, s'emparant de cette question cruciale des «langages de l'art », dont il est certain qu'elle n'a pas fini de nous faire parler.

Yaël Kreplak 


\section{Annexes}

\section{Conventions de transcriptions (ICOR 2007)}

[ ] notent le début et la fin du chevauchement entre deux locuteurs

= note un enchaînement rapide entre deux tours de parole

\& note la continuation du tour par le même locuteur

(o.6) note la durée de la pause

: note des allongements syllabiques

- note la troncation d'un mot esquissé et `note une élision non standard

I et \ notent les montées et descentes intonatives

le soulignement note une insistance, les MAJUSCULES notent une hausse du volume de la voix, tandis que oles degrés ${ }^{\circ}$ notent une baisse

Exemple 1, white cube :

1 JOH D'Allleurs c'est ça qu’ est super drôle c'est c'qui est

2 arrivé avec euh: (0.5) cette pièce là euh: (0.7) du

3 white cube c'est encore un autre rebondiss 'ment $\backslash(0.9)$

4 mais euh c'est marc domage qu'a fait les photos euh pour

5 l'expo au palais de tokyo/ (o.8) et euh: chaque année le

6 palais de tokyo édite un cata[logue sur toute la\&

7 LIL [oui

$8 \mathrm{JOH}$ \&programmation annuelle $\backslash$ donc ils ont aussi documenté

9 mon- mon module (o.6) et en fait (pour moi)

10 l'exposition

11 y a des i- $d$ - des livres qui ont été volés (.) dans ma

12 pièce et donc ils ont fait des photos pour l' constat

13 d'assurance (o.8) et au moment où ils ont publié le: le

14 truc annuel ${ }^{\circ} \mathrm{en}$ fai $\mathrm{t}^{\circ}$ palais de tokyo ils [ONT\&

[ouais

$16 \mathrm{PAU}$

$[\mathrm{xx}$

$17 \mathrm{JOH}$ \&INTERVERti les photos de marc domage avec celles faites

18 (.) pour l'assurance c' qui fait que dans I' bouquin

19 palais de tokyo en fait ce qui est reproduit c'est la

20 pièce sur- où les photos [sont super moches avec les\&

$21 \mathrm{PAU}$

[avec un livre sorti

$22 \mathrm{JOH}$ \&bouquins qui manquent (0.5) à l'intérieur donc à partir

23 de ç[a j'ai fait une nouvelle pièce

24 LIL [une erreur bah oui une erreur UNE ERREUR PAREILLE ÇA

25 NE SE LOUPE PAS/=

$26 \mathrm{JOH}=<(($ en riant $))$ donc $\mathrm{j}$ 'ai fait une nouvelle pièce après> 
27 qui à partir de la reproduction de la page $d$ - du du

28 catalogue quoi qu'était pas la bonne reproduction (0.4)

29 mais enfin enfin là c'est encore des histoires de de de

30 mauvaise (o.6) de mauvaise (.) de mauvaise traduction

$31 \quad{ }^{\circ}$ ('fin) de quelque chose ${ }^{\circ}[(0.6)$ mais c'est c- en fait\&

32 LIL [ouais:

$33 \mathrm{PAU}$

[mmmh

$34 \mathrm{JOH}$ \& c'est vraiment c- c' qui m'intéresse le plus quoi c'est

comme la pièce avec le: (o.4) ${ }^{\circ}$ le: (une) les deux

36 chaises de kosuth là où t'[as:

37 LIL

38 PAU

[oui: il man[que

$39 \mathrm{JOH}$

[ouais ouais

40

41

42

43

44

45

46 LIL ${ }^{\circ 0} \mathrm{mmh} \mathrm{mmh}{ }^{\circ 0}$

47

(.)

$48 \mathrm{JOH}$ alors ils étaient super confus: euh ils m’ont envoyé une

49 lettre d'excuses ${ }^{\circ}$ des trucs comme ça ${ }^{\circ} \mathrm{xx}$ non non c'est

50 très bien

51 LIL [(rires)

52 PAU [(rires)

$53 \mathrm{JOH} x x$ [x quand ça peut

54 PAU [(merci) du terreau pour [euh pour l'avenir

$55 \mathrm{JOH}$

[non mais voilà c'est plutôt

$56 \mathrm{LIL} \mathrm{mmh}$

$57 \mathrm{JOH}$ plutôt en fait tout c' qui peut: final'ment nous déplacer

58 nous- nous- nous renvoyer ailleurs (.) ouvrir sur quelque

59 chose d'autre et[:euh c'est là qu’ finalement commence\&

$60 \mathrm{LIL}$

[mmh mmh

$61 \mathrm{JOH}$ \&le travail véritablement [(.) et $j{ }^{\top}$ pense qu’avec la\&

$62 \mathrm{LIL}$

[mmh

$63 \mathrm{JOH}$ \&situation d' l'expo-sition: ici et puis du fait de

64

65 LIL pouvoir travailler dans une certaine durée/ [(.) ça- ça-\&

$66 \mathrm{JOH}$

[mmh

67 d'approfondir c- c- cette chose là qui est ce qui

68 m'intéresse le plus en fait ${ }^{\circ}$ d[ans: dans le travail\&

69 LIL

[mmh 


\section{$70 \mathrm{JOH}$ [\&quoi}

$71 \mathrm{PAU}[\mathrm{mmh}$

72 LIL et est-ce que tu penses que: on peut définir un peu

Exemple 2, hamilton :

1 ARN ${ }^{\circ}$ (peut-être pas celles qui sont là) ${ }^{\circ}$ et donc la question

2 posée c'est justement peut-on encore regarder après les

3 affaires dutroux et autres choses de ce genre $(0.9)$

4 alors (1.3) hier j'ai eu un groupe un peu plus âgé qui:

5 en discu- c'est vrai qu'à l'époque (.) ça nous choquait

6 pas et maint 'nant on est plus:

7 JEA (mais ; bah) oui mais parce que l'information n'était\&

8 ELI bah oui

9 JEA \&pas la même (.) $x x$ d'inceste de viol étaient moins [(.)\&

10 ARN [oui

11 JEA \&disons (dans\& \&le domaine public) que [xx

$12 \mathrm{ELI}$

13 (.) un regard vers un: un j- un jeune nu n'était pas

14 considéré comme un regard pervers parce que maint 'nant

15 c'est considéré comme pervers $\mathrm{xx}=$

16 ARN $=$ d'ac[cord

17 JEA [oui oui voilà=

$18 \mathrm{ELI}$ =un homme regarde une jeune femme (.) de quinze ans (.)

19 on voit tout de suite du pervers à l'époque on voyait la

20 beauté c'est tout

21 ARN mmh (.) c' qui est amusant c'est que j'ai un[:

$22 \mathrm{ELI}$

['fin c'est

23

24 JEA [oui

25 ARN haha ${ }^{\circ}<\left((\right.$ en riant)) $)$ les collections en question ${ }^{\circ}>($.) euh:

26 I- lorque je viens avec des des des générations plus

27 jeunes (.) j'ai eu un un uni- universitaire qui est

28 arrivé ici que avec $\mathrm{xx}<($ (change de voix)) ouais:> $\mathrm{j}$ ' lui

29 ai dit l'âge il a dit c'est dégueulassel

$30 \quad(1.4)$

31 ((COM: plusieurs visiteurs prennent la parole en même

32 temps))

33 ? et vous lui auriez pas dit l'âge il aurait [rien dit

34 ARN

[bah

exactement et en plus il doit avoir dix neuf ans (.) donc

$37 ?$

[(inaud.) 
Exemple 3, turkish artist :

1 MIK this ey: i come from albany in australia/

2 euh can you speak english//

3 MAR i can [i can slowly]

4 MIK [(inaud.) ] so i come from albany

5 in australia\originally but not from a

6 long 'time $\mathrm{a}\left[\mathrm{go}^{\circ} \backslash\right.$ (.) but (this ; there's

7 MAR $\quad\left[{ }^{\circ}\right.$ yeah $^{\circ}$

8 MIK a) very beautiful artist from albany he's

9 a turkish artist (0.3) who made a work

10 about bee:s but this really reminds me (of

11 him) (0.3) he made it:/ (.) at the end of

12 the eighties $\backslash$ (.) he committed suicide

13 about five years ago ${ }^{\circ}$ (it's sad) ${ }^{\circ}$ but (.)

14 i'll t- i'd lik- i'll try to find a

15 picture of it for you cause he really

16 worked a beautiful work you know and $\mathrm{i}$

17 have to: (.) to show it to you ${ }^{\circ}$ cause it's

18 really ${ }^{\circ}$

19 (2.5)

20 MIK no it's great 